\title{
Pair creation at shocks: Application to the high energy emission of compact objects
}

\author{
P. O. Petrucci ${ }^{1}$, G. Henri ${ }^{2}$, and G. Pelletier ${ }^{2}$ \\ 1 Osservatorio Astronomico di Brera, via Brera 28, 20121 Milano, Italy \\ 2 Laboratoire d'Astrophysique, Observatoire de Grenoble, BP 53X, 38041 Grenoble Cedex, France
}

Received 19 December 2000 / Accepted 27 April 2001

\begin{abstract}
We investigate the effect of pair creation on a shock structure. Particles, accelerated in the shock via the first order Fermi process, are supposed to cool by the inverse Compton process on external soft photons, resulting in a cut-off power law shape of the particle distribution function. The high energy photons produced are thus able to create pairs, through photon-photon annihilation. The increase of the pair pressure may then be sufficient to modify the shock profile. We show that there is even a limit of the pair pressure (of the order of $20 \%$ of the ram pressure of the upstream flow) above which the shock can no longer exist. Conversely, significant changes of the flow velocity profile will also modify the spectral index and the high energy cut-off of the particle distribution function. Hence the number of particles able to trigger the pair creation process will change, modifying the pair creation rate accordingly. Taking into account these different processes, we self-consistently derive the flow velocity profile and the particle distribution function. We show that, in some regions of the parameter space, the system can converge towards stationary states where pair creation and hydrodynamical effects balance. We discuss the application of this model to explain the high energy emission observed in compact objects. We show that hard X-ray spectra $\left(\alpha_{\mathrm{X}}<1\right.$.) are only obtained for low pair pressures and we don't expect any strong annihilation line in this case. We also suggest a possible variability mechanism if the soft photon compactness itself depends on the pair density of the hot plasma, such as is expected in reillumination models.
\end{abstract}

Key words. acceleration of particles - radiation mechanisms: non-thermal - shock waves - X-rays: general

\section{Introduction}

It is well-known that particles can be accelerated up to very high energies by crossing a magnetized shock front many times and thus experiencing the so-called first order Fermi process (Axford et al. 1976; Krimsky 1977; Bell 1978; Blandford \& Ostriker 1978). The simple linear theory may however be insufficient to describe a realistic situation because the accelerated particles may induce a strong non-linear back reaction on the shock itself. For example, they may produce magnetohydrodynamic (Jokipii 1976; Lacombe 1977) or electrostatic (Nishikawa et al. 1994) instabilities, and also strongly alter the hydrodynamics of the shock through their own pressure (Drury et al. 1982; Schneider \& Kirk 1987).

In the case of astrophysical objects like the Active Galactic Nuclei (herafter AGNs), where shocks are particularly attractive processes for the production of in situ high energy particles, the presence of important radiation fields requires the treatment of particle-photon interac-

Send offprint requests to: P. O. Petrucci,

e-mail: petrucci@brera.mi.astro.it,

petrucci@obs.ujf-grenoble.fr tions in the shock region. A large number of works have already studied particle acceleration at shocks including radiative cooling processes (Webb et al. 1984; Schlickeiser 1984; Bregman 1985; Heavens \& Meisenheimer 1987; Biermann \& Strittmater 1987; Protheroe \& Stanev 1999; Drury et al. 1999) or the feedback of the accelerated particle pressures on the shock structure (Blandford 1980; Drury \& Volk 1981; Heavens 1983).

The aim of this paper is to investigate also the effect of pair creation on the shock structure. Indeed particles accelerated by the shock can be sufficiently energetic to boost, via for example the Inverse Compton (hereafter IC) process, the surrounding external soft photons above the rest mass electron energy, thus allowing the triggering of the pair creation process. The increase of the associated pair pressure is thus able to disrupt the plasma flow and eventually, for too high a pressure (due to catastrophic run-away pair production for example), to smooth it completely. Reversely, significant changes of the flow velocity profile may modify the distribution function of the accelerated particles in such a way that the number of particles 
able to trigger the pair creation process can change, thus modifying the pair creation rate.

This non-linearity of the problem occurs in addition to the intrinsic non linearity of the pair creation process, which may be more or less important depending on the compactness of the soft and hard photon field in the source (Svensson 1987). The pair creation process is also naturally non-local in the sense that pairs are produced by photons which are themselves emitted by particles located in another part of the plasma. The complete 3D-treatment of this problem is beyond the scope of this paper. We propose, however, to use simplifying assumptions in 1Dgeometry to study the global behavior of a shock structure in an environment dominated by pairs.

This paper is divided as follows. We first present in Sect. 2 the basic hypotheses we use to treat (relatively easily) the problem without loss of generality. Some important definitions and descriptions of the geometry of our model are detailed in Sect. 3. We then present the main equations describing the behavior of a shock in a pair environment in Sect. 4. In Sect. 5, we first discuss the case without hydrodynamical feedback on the pair process, deducing a limit of the pair creation rate above which the shock disappears. When feedback is taken into account, we study in Sect. 6 the existence of stationary states where pair creation and hydrodynamical effects balance. We discuss the results in Sect. 7 before concluding.

Throughout the paper, upstream and downstream quantities are denoted by subscripts 1 and 2 respectively. Particle and photon energies are expressed (unless pointed out) in units of $m_{\mathrm{e}} c^{2}$, speeds in units of $u_{1}$, the upstream flow velocity, lengths in units of the upstream diffusion length $D_{1} / u_{1}$, where $D_{1}$ is the upstream diffusion coefficient, and time in units of $D_{1} / u_{1}^{2}$. Variables in reduced units are overbraced by $\mathrm{a} \sim$.

\section{Basic hypotheses}

\subsection{Simple geometry}

We assume a 1D geometry (the $x$ axis being aligned with the flow velocity cf. Fig. 1) meaning that the different parameters characterizing the flow are homogeneous in each section perpendicular to the $x$ axis. However, we will use the transverse shock radius $R$ to evaluate the size of the pair creation region $R_{\gamma \gamma}$ in Sect. 3.2.2. The 1-D assumption is certainly well justified in the central part of the flow where boundary effects are negligible. We also make the assumption of a parallel shock, i.e. the magnetic field lines are supposed to be parallel to the normal of the shock front. We however assume the magnetic field to be slightly perturbed near the shock and we suppose these perturbations to be dominated by Alfven waves. It is thus possible for particles to be scattered by these waves through pitch angle scattering (Jokipii 1976; Lacombe 1977) and thus going back and forth across the shock.

\subsection{Constant coefficient diffusion}

Rigorously, the upstream and downstream spatial diffusion coefficients, $D_{1, x x}$ and $D_{2, x x}$, depend slightly on $\gamma$. For small Larmor radii of the particles compared to the large scale of the turbulence (which is well-verified for leptons), one can show (Casse et al. 2001) that $D_{i, x x} \propto \gamma^{2-\beta}$ where $\beta=5 / 3$ for a Kolmogorov spectrum. The dependence on $\gamma$ being weak, we then consider $D_{i, x x}$ to be independent of the Lorentz factor $\gamma$ of the particles. We also assume $D_{i, x x}$ to be independent of $x$. Since we work in 1D-geometry, the upstream and downstream diffusion coefficients will be simply noted $D_{1}$ and $D_{2}$ respectively in the following.

\subsection{Plasma dominated by the relativistic pressure}

We suppose the plasma pressure $P_{\text {tot }}$ to be dominated by the pressure $P_{\text {rel }}$ of the relativistic lepton population. This seems reasonable since the lepton acceleration time scale is expected to be relatively small (and significantly smaller than the proton acceleration time scale, Henri et al. 1999). $P_{\text {rel }}$ thus rapidly becomes larger than the thermal pressure. In this case, the flow velocity profile already makes a smooth transition between the up and downstream region but acceleration still occurs (Drury et al. 1982; Schneider \& Kirk 1987). Besides, with this assumption, the specific heat ratio of the accelerated particle plasma is equal to $4 / 3$. Consequently, in the case of a strong shock, the compression ratio reaches the value of 7 .

\subsection{Assuming a large Alfvenic Mach number}

Although the magnetic field is necessary to produce the stochastic acceleration, we will assume that the Alfven velocity $V_{\mathrm{A}}=B / \sqrt{\rho}$ (where $B$ is the magnetic field amplitude and $\rho$ the plasma density) is much smaller than the upstream flow velocity $u_{1}$ i.e. the Alfvenic Mach number $M_{\mathrm{A}}=u_{1} / V_{\mathrm{A}}$ is very large. This leads to important simplifications in the treatment of the acceleration process (Vainio \& Schlikeiser 1998, 1999; Schlickeiser et al. 1993; Henri et al. 1999):

- the second order Fermi process is negligible compared to the first order one, since the ratio of the acceleration time scale is approximately $t_{1 \mathrm{st}} / t_{2 \mathrm{nd}} \simeq M_{\mathrm{A}}^{-2}$

- The scattering center compression ratio (which controls the particle distribution spectral index) is close to the gas compression ratio $r=u_{1} / u_{2}$

Consequently, in the following, we will neglect the second order Fermi process in our treatment and we will only use the gas compression ratio for the computation of the particle distribution function (cf. Sect. 3.3).

\subsection{Compton cooling in the Thomson regime}

We assume the particle cooling to be dominated by the Inverse Compton process onto the soft photons, neglecting 


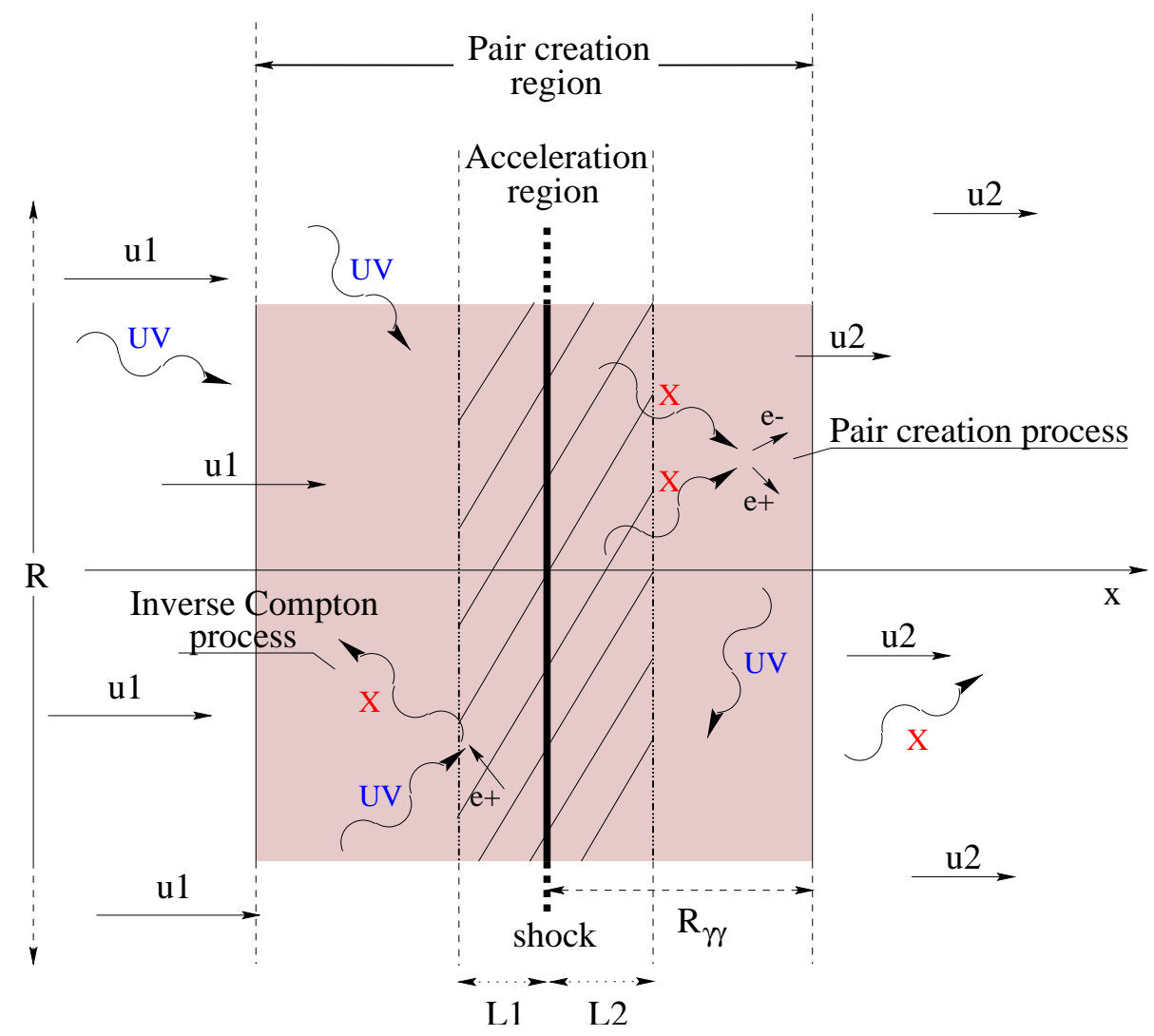

Fig. 1. Schematic view of a shock. The shock discontinuity is represented by the vertical bold line. We have also indicated the different parameters defining the acceleration and pair creation region (cf. Sect. 3). The acceleration region is the region in between the 2 vertical dot-dashed lines. The pair creation region is filled in grey. Particles are represented by straight arrows and photons by warped ones. The diagram is not drawn to scale.

the synchrotron process. Again it is justified in the case of low magnetic energy density. For simplicity, the external soft photon field is supposed to be monoenergetic and we note $\epsilon_{\mathrm{s}}$, the corresponding soft photon energy. This is not a bad approximation if the soft photon field is emitted by a thermal plasma, like that probably associated with the Blue Bump emission seen in most AGNs. Furthermore, we will restrict our study to the Thomson regime i.e. $\epsilon_{\mathrm{s}} \gamma_{\mathrm{c}}<1$ where $\gamma_{c}$ is the high energy cut-off Lorentz factor of the particle distribution (cf. Sect. 3.3.3).

\section{Some important parameters and definitions}

\subsection{The acceleration process}

\subsubsection{The acceleration threshold}

Only particles having a Larmor radius $r_{\mathrm{L}}$ comparable to a wavelength of the Alfven spectrum will undergo scattering (Jokipii 1976; Lacombe 1977) and thus will go back and forth many times across the shock. However, in $e^{-}-p^{+}$ plasma, such as those we deal with, the non-relativistic protons limit the Alfven wave spectrum to wavelengths greater than $2 \pi V_{\mathrm{A}} / \omega_{\mathrm{cp}}$ where $\omega_{\mathrm{cp}}$ is the cyclotron pulsation of the protons in a magnetic field $B$. Thus a particle of momentum $p$ will be scattered by Alfven waves if it satisfies:

$r_{\mathrm{L}}=\frac{p}{|q B|} \geq \frac{m_{\mathrm{p}} V_{\mathrm{A}}}{|q B|}$

that is, $p \geq m_{\mathrm{p}} V_{\mathrm{A}}$. This puts a severe threshold for resonant interactions, especially for the leptons that must be already very energetic to participate into the diffusion process. Indeed, from Eq. (1) we can deduce the lower Lorentz factor for a relativistic lepton to be accelerated in a shock:

$\gamma_{\text {scat }}=\frac{m_{\mathrm{p}}}{m_{\mathrm{e}}} \frac{V_{\mathrm{A}}}{c}$

which could easily be of the order of a few units. Several processes are known to accelerate electrons up to such an energy, such as the development of a parallel electric field component in magnetic reconnections, or waves (magnetosonic or whistler which have a much lower particle energy threshold for resonant interaction, Ragot \& Schlickeiser 1998). We will assume that such processes accelerate the particles up to a characteristic Lorentz factor $\gamma_{\text {min }}$ for which the pre-acceleration and cooling times are equal. We further assume that $\gamma_{\min }$ is above the scattering threshold $\gamma_{\text {scat }}$.

It is worth noting that the high energy power law spectra observed in compact objects generaly extend down to the $\mathrm{keV}$ range. For non-thermal models, it implies that the 
injection threshold is low $\left(\gamma_{\text {scat }}<10\right)$, which is consistent with a small Alfvenic velocity.

\subsubsection{The acceleration region}

Particles interacting with the shock are those located within about one diffusion length of the shock, where the upstream and downstream diffusion length are defined by (as previously said, $i=1,2$ for up and downstream respectively):

$L_{i}=\frac{D_{i}}{u_{i}}$

For a velocity profile distorted by the presence of pairs, this definition should not be rigorously used. However, we expect the change of the velocity in the vicinity of the shock, due to the pair pressure, to be relatively small, of the order of unity (the compression ratio $r=u_{1} / u_{2}$ can only vary between 1 and 7). The region explored by particles will thus have a size not very different from that given by Eq. (3) We will call in the following "acceleration" region the physical space defined by $-L_{1} \leq x \leq+L_{2}$ (cf. Fig. 1).

\subsubsection{The acceleration time scale}

At each back and forth passage across the shock front, a relativistic particle gains energy such that:

$\frac{\delta p}{p}=\frac{4}{3} \frac{u_{1}-u_{2}}{v \cos \theta_{1}}$

where $v$ is the particle velocity and $\theta_{1}$ is the angle of the magnetic field line with respect with the shock normal. In our case (a parallel shock) we assume $\theta_{1}=0$.

On the other hand, the residence time $t_{\mathrm{r}}$ of this particle in the vicinity of the shock is determined by the diffusion coefficient:

$t_{\mathrm{r}} \simeq \frac{D_{1}}{u_{1}^{2}}+\frac{D_{2}}{u_{2}^{2}}$

If the upstream and downstream diffusion coefficients were equal, the downstream contribution would be dominant. However, as shown by Vainio \& Schlickeiser (1998), there is generally an increase of the turbulence level in the downstream flow so that $D_{1}$ is expected to be significantly larger than $D_{2}$. We refrain from using too involved calculations and we assume that $t_{\mathrm{r}}$ is simply of the order of the first term, i.e. $t_{\mathrm{r}} \simeq \frac{D_{1}}{u_{1}^{2}}$.

At each crossing, the particle has a probability $\eta \simeq$ $\frac{4 u_{2}}{v}$ of escaping (Bell 1978) and for a relativistic particle the crossing frequency is roughly $\nu_{\mathrm{c}} \simeq \frac{1}{\eta t_{\mathrm{r}}}$. The acceleration rate is therefore (with a factor of the order of unity):

$\frac{\langle\Delta p\rangle}{p \Delta t} \simeq \frac{r-1}{3 t_{\mathrm{r}}}$
We can thus deduce the corresponding acceleration time scale:

$$
t_{\mathrm{acc}} \simeq 3 \frac{D_{1}}{u_{1}^{2}} \frac{1}{r-1}=\frac{D_{1}}{u_{1}^{2}} \widetilde{t}_{\mathrm{acc}}
$$

\subsection{The pair creation process}

\subsubsection{The pair creation threshold}

A soft photon of energy $\epsilon_{\mathrm{s}}$ (in reduced units) scattered by a lepton of Lorentz factor $\gamma$ will be boosted, via the IC process, to an energy $\epsilon \simeq \frac{4}{3} \gamma^{2} \epsilon_{\mathrm{s}}$. The high energy photon produced will be able to give an electron-positron pair if at least :

$\epsilon \gtrsim 1$ i.e. $\gamma \gtrsim\left(\frac{3}{4 \epsilon_{\mathrm{s}}}\right)^{1 / 2}=\gamma_{\mathrm{th}}$.

A particle needs thus to have a Lorentz factor higher than $\gamma_{\text {th }}$ to generate an electron/positron pair. To fix an order of magnitude, for a UV bump peaking near $10 \mathrm{eV}$ (a common value in AGNs, Walter et al. 1994), Eq. (5) gives $\gamma_{\text {th }} \simeq 300$. We will suppose that $\gamma_{\min } \leq \gamma_{\text {th }}$ so that particles need to be accelerated in the shock to be able to trigger the pair creation process.

\subsubsection{The pair creation region}

If the shock region is compact enough and if part of its radiation extends beyond $511 \mathrm{keV}$, the pair creation, through photon-photon annihilation, will become important. The pair creation optical depth $\tau_{\gamma \gamma}$ depends on photon energy $\epsilon$. Photons with $\epsilon>2$ produced in Compton scatterings will produce pairs mostly with a Lorentz factor $\gamma \simeq \epsilon / 2$ by colliding with photons with energies of the order of $2 / \epsilon$. Consequently, the optical depth for $\epsilon>2$ can be written as follows (Zdziarski \& Lightman 1985):

$$
\begin{aligned}
\tau_{\gamma \gamma}(\epsilon) & \simeq \frac{2}{3} \sigma_{\mathrm{T}} R \frac{n_{\mathrm{ph}}\left(\frac{2}{\epsilon}\right)}{\epsilon} \\
& =\frac{R}{L_{\epsilon}}
\end{aligned}
$$

where $n_{\mathrm{ph}}(\epsilon)$ is the density of photons with energy $\epsilon$ per unit volume and dimensionless energy $\epsilon$, and $L_{\epsilon}=$ $\left(\frac{2}{3} \sigma_{T} \frac{n_{\mathrm{ph}}\left(\frac{2}{\epsilon}\right)}{\epsilon}\right)^{-1}$ is the typical attenuation length of a photon with an energy $\epsilon$. Since we expect the photon density to decrease with energy, $L_{\epsilon}$ is a decreasing function of $\epsilon$ and thus $L_{\epsilon}<L_{o}$ where we define:

$L_{o}=L_{\epsilon=1}$.

On the other hand, the photon density will suffer from geometrical dilution after some distance comparable to $R$, the transverse size of the shock (cf. Fig. 1). Hence, we will define the pair creation region as the region included between $-R_{\gamma \gamma}$ and $+R_{\gamma \gamma}$, where $R_{\gamma \gamma}$ is defined by:

$R_{\gamma \gamma}=\frac{R L_{\mathrm{o}}}{R+L_{\mathrm{o}}}$ 
This definition has the advantage of taking into account both photon-photon depletion and geometrical dilution effects. In the following, we will suppose, for sake of simplicity, that the pair creation rate is constant in this region.

\subsection{The flow profile and particle distribution}

\subsubsection{The "effective" compression ratio}

The definition of the compression ratio as the ratio between the far upstream and downstream flow velocity is rather unsatisfactory in our case since it will not give a real estimate of the velocity change experienced by a relativistic particle in the vicinity of the shock, where the particle distribution is principally built. We will thus define an "effective" compression ratio, noted simply $r$, as the ratio between the upstream velocity in $-10 L_{1}$ and the downstream velocity in $+10 L_{2}$. We have checked that in the case of a strong shock without pairs, this definition still gives a compression ratio very near the expected value of 7 .

\subsubsection{The spectral index}

It can be shown that the solution of the evolution equation of the particle distribution function including the pair creation process (cf. Eq. (13)), still has an energy power law dependence, as it is effectively the case without pairs (cf. Appendix). The spectral index $s^{1}$ also keeps the same expression as a function of the compression ratio i.e.:

$s(r)=q-2=(r+2) /(r-1)$.

For plasma dominated by relativistic pressure, the compression ratio is necessarily smaller than 7 so that $s(r)$ is larger than 1.5.

In fact, Eq. (9) is really valid for not too large a relativistic pressure in comparison to the thermal one $\left(P_{\text {rel }} / P_{\text {th }}\right.$ must be smaller than $\sim 10$, cf. Pelletier \& Roland $1984)$ and we assume we are in this limiting case so that we can also still neglect the thermal pressure of the plasma in comparison to the relativistic one.

\subsubsection{The high energy cut-off}

Since we take into account the cooling, a high energy cutoff in the particle distribution must necessarily appear at a Lorentz factor $\gamma_{\mathrm{c}}$ where heating and cooling balance (Webb et al. 1984). Since we suppose that particles cool via the inverse Compton process, and assuming that the soft photon density is homogeneous in the shock region, the mean cooling time scale can be written:

$t_{\text {cool }}=\frac{3}{4} \frac{m_{\mathrm{e}} c}{\gamma \sigma_{T} W_{\text {soft }}}=\frac{3}{4} \frac{1}{l_{\mathrm{s}} \gamma} \frac{u_{1}}{c} \widetilde{R} \frac{D_{1}}{u_{1}^{2}}$

\footnotetext{
${ }^{1}$ here $s$ is the spectral index of the spatially integrated distribution function of the particles $n(\gamma) \propto \gamma^{-s}$. It is related to the spectral index $q$ defined in Appendix by $s=q-2$.
}

where $l_{\mathrm{s}}$ is the local soft compactness $l_{\mathrm{s}}=\frac{L_{\mathrm{soft}} \sigma_{T}}{4 \pi R m_{\mathrm{e}} c^{3}}, m_{\mathrm{e}}$ being the electron mass, $L_{\text {soft }}$ the soft radiation luminosity injected into the shock region and $W_{\text {soft }}$ the corresponding local soft photon energy density. The maximum Lorentz factor $\gamma_{c}$ achievable by the acceleration process is thus obtained by setting $t_{\mathrm{acc}}=t_{\text {cool }}$ which gives, using Eqs. (4) and (10):

$$
\gamma_{\mathrm{c}}=\frac{\widetilde{R}}{l_{\mathrm{s}}} \frac{u_{1}}{c} \frac{r-1}{4}
$$

\section{Basic kinetic equations at shocks}

The shock region is depicted in Fig. 1 (not to scale) with the acceleration and pair creation region described above. We take the origin of the $x$-axis at the point where the second derivative of the flow velocity vanishes, that is:

$$
\left.\frac{\partial^{2} \widetilde{u}}{\partial \widetilde{x}^{2}}\right|_{\widetilde{x}=0}=0
$$

As previously said, we suppose the existence of a magnetic field B, slightly perturbed by Alfven waves. The particles are thus scattered by these waves through pitch angle scattering (Jokipii 1976; Lacombe 1977) and can cross the shock front several times before escaping unless they are rapidly cooled by radiative processes. During these scatterings, the particles gain energy through the well-known first-order Fermi process. We also suppose the magnetic perturbations to have sufficiently small amplitudes so that we can treat the problem in quasilinear theory using the Fokker-Planck formalism. We also assume that, in each part of the shock front, the scattering is sufficient for the particle distribution to be nearly isotropic. With these different assumptions, and when the first order Fermi process such as radiative losses and pair creation/annihilation are taken into account, the particle distribution function $f(p, x)$ must verify the following equation:

$$
\begin{aligned}
\frac{\partial f}{\partial t}+u \frac{\partial f}{\partial x}= & \frac{1}{3} \frac{\partial u}{\partial x} p \frac{\partial f}{\partial p}+\frac{1}{p^{2}} \frac{\partial b p^{4} f}{\partial p}+\frac{\partial}{\partial x} D \frac{\partial f}{\partial x} \\
& +\mathbf{A}(p)+\mathbf{B}_{ \pm}(p)+\mathbf{C}_{ \pm}(p) .
\end{aligned}
$$

The three first terms of the right member correspond to the first order process, the radiative losses $(b$ being $>0$, in the case of Compton cooling in the Thomson regime $b=1 /\left(\gamma t_{\text {cool }}\right)$ where $t_{\text {cool }}$ is given by Eq. (10)) and the spatial diffusion respectively, $\mathbf{A}(p)$ is the injection of particles at $\gamma_{\min }$ due to the pre-acceleration processes (cf. Sect. 3.1.1), $\mathbf{B}_{ \pm}(p)$ is the pair creation rate and $\mathbf{C}_{ \pm}(p)$ the annihilation one. This equation is essentially the equation of the cosmic-ray transport originally given by Parker (1965) and Skilling (1975 and references therein) except for the addition of the radiative, pre-acceleration and pair processes. 


\subsection{Evolution equation of the flow velocity profile}

As previously stated in Sect. 2, we supposed the shocked plasma pressure to be dominated by the pressure of the relativistic particles. The latter can thus be written as follows:

$P_{\text {rel }}=\int 4 \pi p^{2} \frac{p c}{3} f(p, x) \mathrm{d} p$.

Consequently, by multiplying Eq. (13) by $4 \pi p^{3} c / 3$ and integrating over $p$ we obtain the hydrodynamic equation linking pairs (through their pressure) and the flow velocity that is (in the stationary state):

$u \frac{\partial P_{\mathrm{rel}}}{\partial x}+\frac{4}{3} P_{\mathrm{rel}} \frac{\partial u}{\partial x}=\frac{\partial}{\partial x} D \frac{\partial}{\partial x} P_{\mathrm{rel}}+\dot{Q}^{-}+\dot{P}_{ \pm}+\dot{Q}^{+}$

where:

$\dot{Q}^{-}=\int 4 \pi \frac{p c}{3} \frac{\partial b p^{4} f}{\partial p} \mathrm{~d} p$

corresponds to the pressure loss rate due to radiative losses (it is thus obviously negative),

$\dot{Q}^{+}=\int \frac{4 \pi p^{3} c}{3} \mathbf{A}(p) \mathrm{d} p$

is the pair pressure creation rate due to the pre-accelerator processes, and

$\dot{P}_{ \pm}=\int \frac{4 \pi p^{3} c}{3} \mathbf{B}_{ \pm}(p) \mathrm{d} p+\int \frac{4 \pi p^{3} c}{3} \mathbf{C}_{ \pm}(p) \mathrm{d} p$

is the pressure creation rate including the pair creation and annihilation processes. It is now possible to deduce from Eq. (14) the differential equation followed by the flow velocity. Indeed the momentum conservation equation gives:

$\rho\left(\frac{\partial u}{\partial t}+u \frac{\partial u}{\partial x}\right)+\frac{\partial P_{\text {rel }}}{\partial x}=0$

which, in the stationary state, is easily integrated:

$\rho u^{2}+P_{\text {rel }}=\mathcal{C}$

where $\mathcal{C}$ is a constant. Assuming that the mass is dominated by protons (i.e. $\rho=n_{\mathrm{p}} m_{\mathrm{p}}$ ) and thus is conserved, Eq. (15) gives:

$P_{\text {rel }}=\rho_{1} u_{1}\left(u_{1}-u\right)$.

To obtain this equation we have supposed $\left.P_{\text {rel }}\right|_{-\infty}=0$ and $\left.u\right|_{-\infty}=u_{1}$. Then Eq. (14) finally gives:

$\frac{4}{3} \frac{\partial \widetilde{u}}{\partial \widetilde{x}}-\frac{7}{3} \widetilde{u} \frac{\partial \widetilde{u}}{\partial \widetilde{x}}+\frac{\partial^{2}}{\partial \widetilde{x}^{2}} \widetilde{u}=\frac{\partial}{\partial \widetilde{x}}\left(\frac{4}{3} \widetilde{u}-\frac{7}{6} \widetilde{u}^{2}+\frac{\partial \widetilde{u}}{\partial \widetilde{x}}\right)=\frac{\Pi}{\widetilde{R}_{\gamma \gamma}}(16)$

where the reduced variables are defined as followed:

$$
\begin{aligned}
\widetilde{u} & =\frac{u}{u_{1}} \\
\widetilde{x} & =\frac{x}{L_{1}} \text { and } \widetilde{R}_{\gamma \gamma}=\frac{R_{\gamma \gamma}}{L_{1}} \\
\Pi & =\frac{\dot{Q}^{+}+\dot{P}_{ \pm}+\dot{Q}^{-}}{\rho_{1} u_{1}^{3}} R_{\gamma \gamma} .
\end{aligned}
$$

The parameter $\Pi$ is the ratio of the flux of energy transmitted to particles, through pair creation/annihilation, pre-acceleration and cooling processes, integrated on the "1D" volume $\widetilde{R}_{\gamma \gamma}$, to the flux of kinetic energy of the upstream flow. As the acceleration and the cooling time are short with respect to the travel time $R_{\gamma \gamma} / c$, we can assume that the sum of the 3 terms $\dot{Q}^{+}, \dot{P}_{ \pm}$, and $\dot{Q}^{-}$of Eq. (19) is equivalent to the instantaneous creation of pairs at the Lorentz factor $\gamma_{\min }$, that is:

$$
\begin{aligned}
\dot{Q}^{+}+\dot{P}_{ \pm}+\dot{Q}^{-} & \simeq \frac{\gamma_{\min } m_{\mathrm{e}} c^{2}}{3} \int 4 \pi p^{2} \mathbf{B}_{ \pm}(p) \mathrm{d} p \\
& =\frac{\gamma_{\min } m_{\mathrm{e}} c^{2}}{3} \dot{n}_{ \pm}
\end{aligned}
$$

where $\dot{n}_{ \pm}$is the total (integrated over the particle Lorentz factor) pair creation rate. Then, $\Pi$ can also be seen as the ratio of the pair luminosity (the pair power density integrated on the "1D" volume $R_{\gamma \gamma}$ ) to the kinetic energy flux of the flow. This parameter will play an important role in the evolution of the shock profile, as we will see in the following. We can anticipate that a value of $\Pi$ of the order of unity will be certainly unfavorable for the formation of the shock. It means that all the kinetic energy of the upstream flow will be dissipated in radiative cooling and the pair creation/annihilation processes.

\section{Solution without feedback}

In this section, we study the solutions of the previous equations as a function of the parameter $\Pi$. We do not consider the feedback of the hydrodynamics on the pair creation process, i.e. $\Pi$ is considered as a fixed free parameter. The complete problem, which must take into account the different processes to find self consistent solutions, will be treated in the next section.

\subsection{A maximal pair creation rate}

A way to solve Eq. (16) is to integrate it between $-\infty$ and $L_{2}$. In this case, we can neglect the cooling during the integration. Indeed, even if for $x \leq-L_{1}$, particles do not interact with the shock, we have assumed that some processes apply and balance coolings so that particles are injected at a minimum Lorentz factor $\gamma_{\min }$ above the resonance threshold $\gamma_{\text {scat }}$. On the other hand, in the shock region (that is, $-L_{1} \leq x \leq L_{2}$ ), we have seen in Sect. 3.3.3 that the coolings are negligible, in comparison to heatings, for particles with a Lorentz factor smaller than $\gamma_{c}$ (the case of the majority of the particles). We can also neglect the annihilation since it occurs mainly at low energy, i.e. for particles with a Lorentz factor $\gamma \simeq 1$ (cf. Coppi \& Blandford 1990), whereas we assume $\gamma \geq \gamma_{\min }>1$. In these conditions, and with the assumption that the pair creation is homogeneous in the region $-R_{\gamma \gamma}<x<+R_{\gamma \gamma}$ 


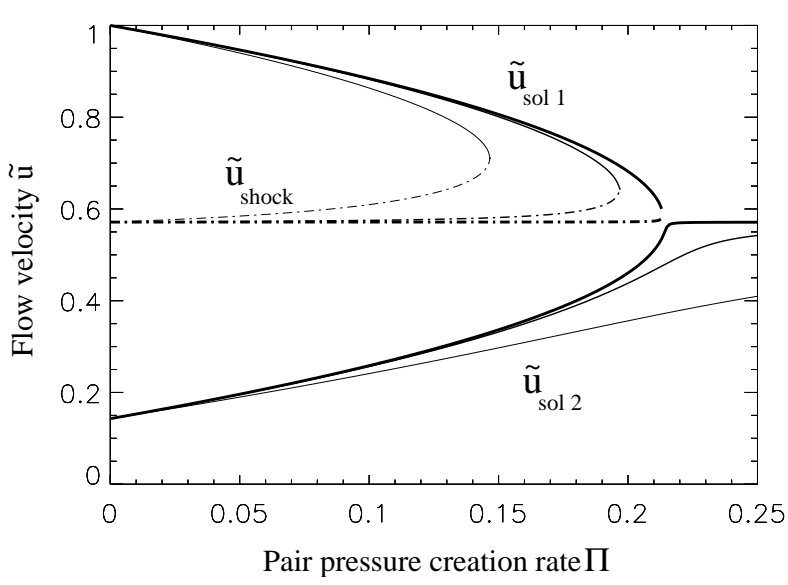

Fig. 2. Plot of the 3 solutions of Eq. (22) versus $\Pi$ for different values of $\widetilde{R}_{\gamma \gamma}$. From thin to thick lines, $\widetilde{R}_{\gamma \gamma}=10,100,10^{3}$. The flow velocity $\widetilde{u}_{\text {shock }}$ at the shock location, i.e. in $\widetilde{x}=0$, is plotted with dashed lines. For each value of $\widetilde{R}_{\gamma \gamma}$, there is a maximal value of $\Pi$, above which only one real solution exists. The disapperance of the $\widetilde{u}_{\text {shock }}$ branch means that the shock cannot exist anymore.

(meaning $\dot{P}_{ \pm}$roughly constant) and null outside (meaning $\dot{P}_{ \pm}=0$ ), the integration of Eq. (16) gives:

$\frac{\partial \widetilde{u}}{\partial \widetilde{x}}=\frac{7}{6}(1-\widetilde{u})\left(\frac{1}{7}-\widetilde{u}\right)+\Pi\left(\frac{\widetilde{x}}{\widetilde{R}_{\gamma \gamma}}+1\right)$.

Equation (21) can be solved with the following boundary conditions:

$\widetilde{u}\left(-\widetilde{R}_{\gamma \gamma}\right)=\widetilde{u}^{*}\left(-\widetilde{R}_{\gamma \gamma}\right)$,

where $\widetilde{u}^{*}$ is the flow velocity solution of Eq. (21) when $\Pi=0$, that is (Drury et al. 1982):

$\widetilde{u}^{*}(x)=\frac{4}{7}-\frac{3}{7} \tanh \left[\frac{2}{7} \int_{x_{0}}^{x} \frac{\mathrm{d} x^{\prime}}{D}\right]$,

and assuming Eq. (12). Using Eq. (21), this last equation becomes:

$$
\begin{aligned}
\left.\frac{\partial^{2} \widetilde{u}}{\partial \widetilde{x}^{2}}\right|_{x=0}=P(\widetilde{u})= & 49 \widetilde{u}^{3}-84 \widetilde{u}^{2}+(39+42 \Pi) \widetilde{u} \\
& -6 \Pi\left(4+\frac{3}{\widetilde{R}_{\gamma \gamma}}\right)-4=0 .
\end{aligned}
$$

This equation possesses in general 3 real solutions which obviously depend on $\Pi$ and $\widetilde{R}_{\gamma \gamma}$. They have been plotted in Fig. 2 as a function of $\Pi$ and for different values of $\widetilde{R}_{\gamma \gamma}$. Of course, if $\Pi$ vanishes, the three solution branches converge respectively to the well-known results $u=1 / 7,4 / 7$ and 1 , corresponding to the values without pair creation. By continuity, the flow velocity at the shock location will follow the second branch noted $u_{\text {shock }}$ on the figure (plotted in dashed line). It appears that, for a given value of $\widetilde{R}_{\gamma \gamma}$, there exists a maximal value $\Pi_{\max }$ of $\Pi$ above which there is only one real solution which still satisfies Eq. (22). The unphysical discontinuity of the flow velocity at the shock location for $\Pi=\Pi_{\max }$ means simply that the shock cannot exist anymore.

We have reported in Fig. 3 the different shapes of the polynomial $P(\widetilde{u})$ and the corresponding flow velocity profiles obtained numerically by solving Eq. (21) for different values of $\Pi$ (fixing $\widetilde{R}_{\gamma \gamma}$ to 10 ). We clearly see the softening of the flow velocity profile when $\Pi$ increases, meaning that the acceleration becomes less and less efficient. We have also plotted in Fig. 4 the variation of the compression ratio $r$ (as defined in Sect. 3.3.1) as a function of the pair creation luminosity $\Pi$ and for different values of $\widetilde{R}_{\gamma \gamma}$. As expected, $r$ converge to $\sim 1$ when $\Pi$ increases.

Since $P(\widetilde{u})$ is a third degree polynomial, the transition between 3 to 1 real solutions happens when the following conditions are satisfied:

$P(u)=0$

$P^{\prime}(u)=0$.

The resolution of this system of equations thus gives a relation between $\Pi_{\max }$ and $\widetilde{R}_{\gamma \gamma}$ :

$\Pi_{\max }=\frac{3}{14}-\frac{1}{14}\left(\frac{63 \Pi_{\max }}{\widetilde{R}_{\gamma \gamma}}\right)^{2 / 3}$.

We have plotted $\Pi_{\max }$ versus $\widetilde{R}_{\gamma \gamma}$ in Fig. 5 . We see that it is an increasing function of $\widetilde{R}_{\gamma \gamma}$, meaning that the larger the pair pressure creation region, the larger the power we need to kill the shock.

But the important conclusion of this section is that $\Pi_{\text {max }}$ is necessarily smaller than $3 / 14 \simeq 0.20$ (cf. Eq. (23)), meaning that at most $20 \%$ of the kinetic energy flux of the upstream flow transformed in pairs is sufficient to suppress the shock discontinuity.

\section{Stationary states}

\subsection{The equations}

At this stage, we have studied the deformation of the flow profile due to pair creation as a function of the parameter П. Conversely, the velocity profile of the flow will control the distribution function of the accelerated particles, and hence the number of particles able to trigger the pair creation process (i.e. particles with $\gamma \geq \gamma_{\text {th }}$ ). This back reaction will thus select the control parameter $\Pi$ defining the possible stationary states for a given set of external parameters.

From the previous section, we have seen that the compression ratio $r$ decreases when the pair creation rate raises (cf. Fig. 4) but conversely if $r$ decreases, the spectral index will increase and the number of high energy particles will decrease (cf. Eqs. (9) and (11)), the final effect being a decrease of the pair creation rate. Consequently, we indeed expect the system to reach, in some conditions, stationary states where hydrodynamics and pair creation effects balance. 

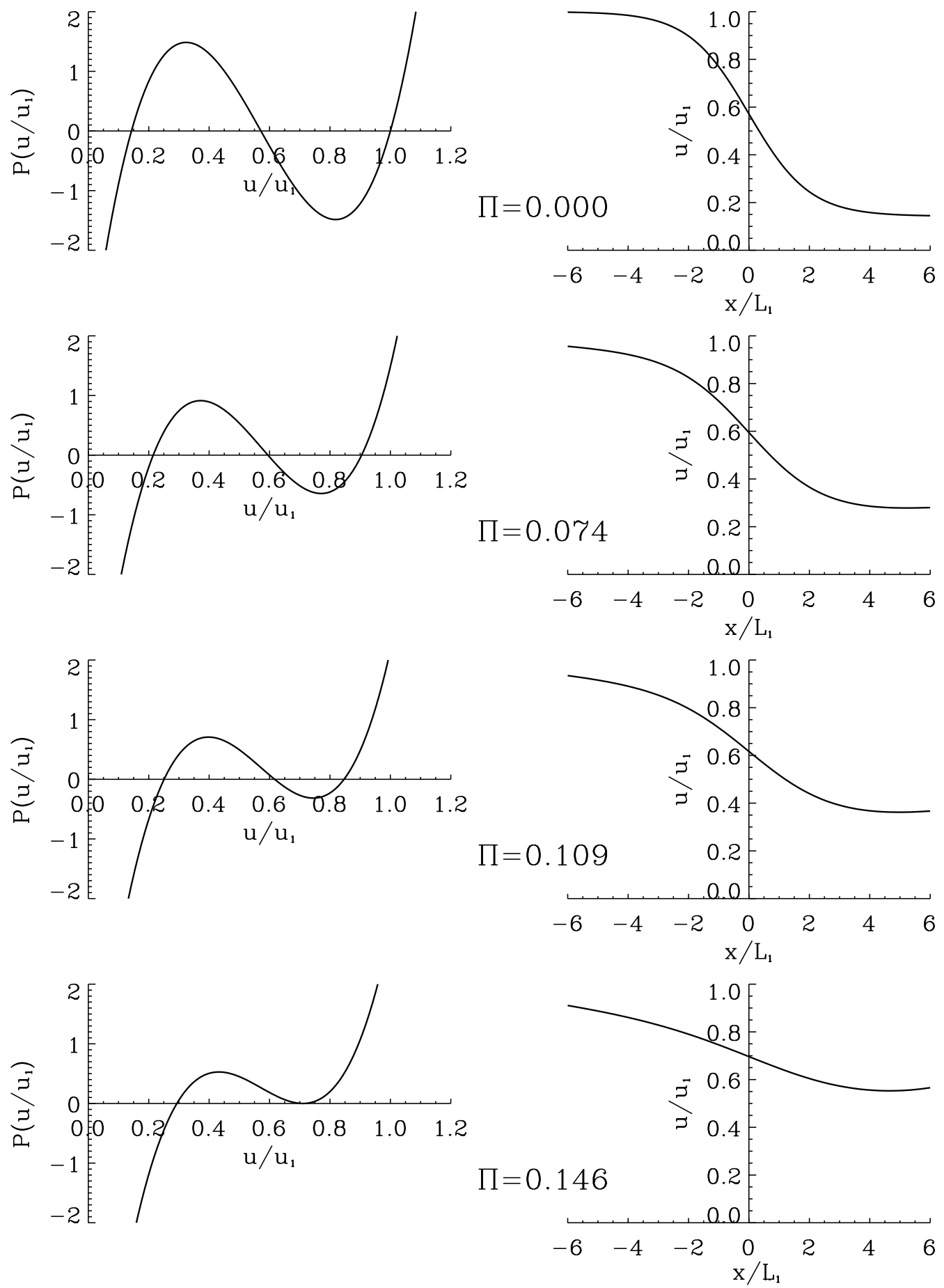

Fig. 3. Disappearance of a shock due to pair creation. We have taken $\widetilde{R}_{\gamma \gamma}=10$ meaning that $\Pi$ must be smaller than $\sim 0.15$ (cf. Eq. (23)).

The problem is now to calculate the pair production rate as a function of the flow profile parameters. Towards this goal, we first approximate the downstream particle distribution function just after the shock with the following form:

$n_{2}\left(\gamma, x=0^{+}\right)=n_{\mathrm{o}} \gamma^{-s} \exp \left(-\frac{\gamma}{\gamma_{\mathrm{c}}}\right)$
From the remarks in Sect. 3.3, it appears to be a relatively good approximation in the vicinity of shocks where the first order Fermi process and Compton coolings occur. The assumption of an exponential high energy cut-off appears also to be reasonnable (cf. for exemple Webb et al. 1984).

The particle energy distribution is then completely determined by $n_{0}, s$, and $\gamma_{\mathrm{c}}$. The latter are functions only of the compression ratio $r$ and the external and geometrical parameters $\widetilde{R}, l_{\mathrm{s}}$ and $u_{1} / c$. We can thus express 


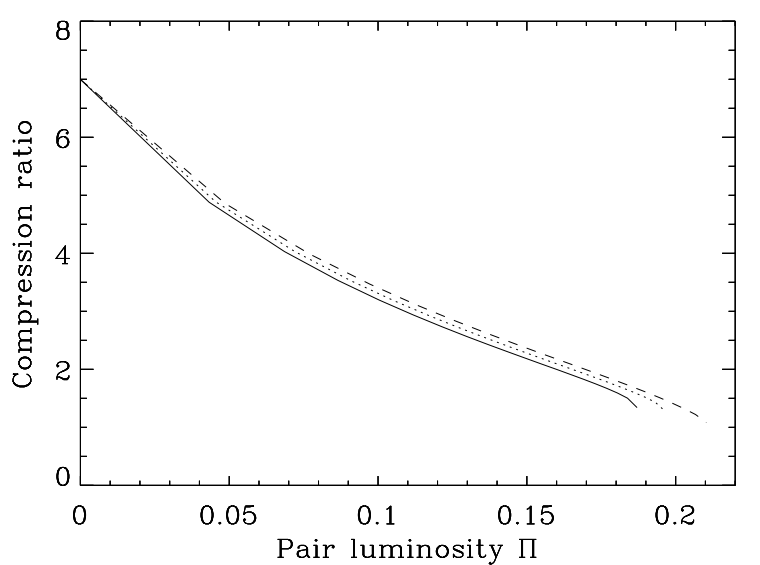

Fig. 4. Variation of the compression ratio $r$ as a function of the pair luminosity $\Pi$ for different values of $\widetilde{R}_{\gamma \gamma}$. $\widetilde{R}_{\gamma \gamma}=10$, 100, 1000 for the solid, dotted and dot-dashed line respectively.

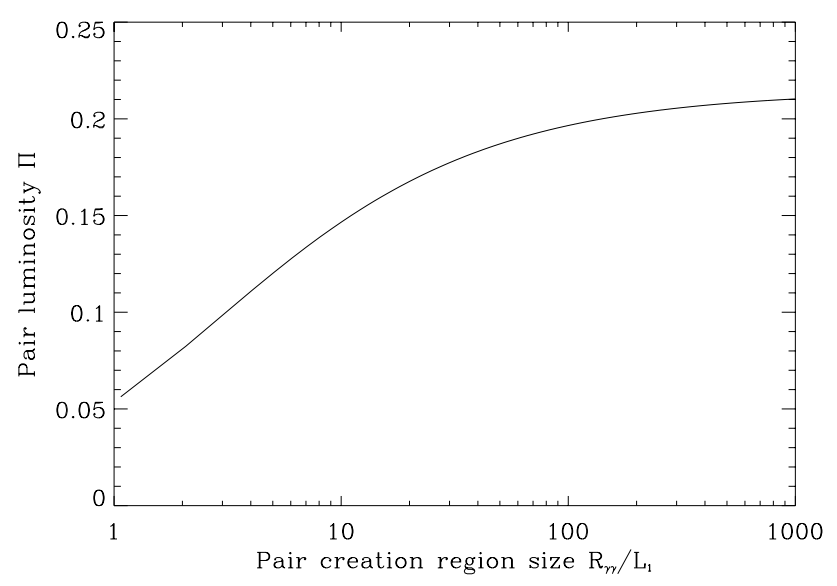

Fig. 5. Plot of the maximal pair luminosity $\Pi_{\max }$ versus the size $\widetilde{R}_{\gamma \gamma}=R_{\gamma \gamma} / L_{1}$ of the pair creation region.

$n_{2}\left(\gamma, x=0^{+}\right)$as a function only of these external parameters and the two dimensionless variables $r$ and

$\tilde{n}_{0}=\frac{m_{\mathrm{e}}}{\rho_{1}} n_{0}$.

The compression ratio $r$ is a function of $\Pi$ and $\widetilde{R}_{\gamma \gamma}$ through the numerical integration of Eq. (21). We can express also $\widetilde{n}_{0}$ as a function of these two quantities in the following way. For simplicity, we will neglect, in the acceleration processes, particles coming from the upstream flow and consider the plasma to be dominated by created pairs. Consequently, assuming that the pair creation rate $\dot{n}_{ \pm}$is constant within the pair creation region, the total downstream particle density $n_{\text {tot }}$ after the shock can be written:

$n_{\text {tot }}=\int_{\gamma_{\min }}^{\infty} n_{2}\left(\gamma, x=0^{+}\right) \mathrm{d} \gamma \simeq \dot{n}_{ \pm} \frac{R_{\gamma \gamma}}{u_{2}}$.

With the help of Eqs. (24) and (25), we thus find:

$\widetilde{n}_{o} \simeq \frac{m_{e}}{\rho_{1}} \dot{n}_{ \pm} \frac{R_{\gamma \gamma}}{u_{2}} \frac{(s-1)}{\gamma_{\min }^{1-s}}$ where we have use the approximation $\int_{\gamma_{\min }}^{\infty} \gamma^{-s} \exp \left(-\frac{\gamma}{\gamma_{c}}\right) \mathrm{d} \gamma \simeq \frac{\gamma_{\min }^{1-s}}{s-1}$, reasonable for $s>1$, which is always our case (cf. Sect. 3.3).

As we assume that the produced pairs are instantaneously accelerated to the Lorentz factor $\gamma_{\min }$, the pair luminosity $\Pi$ can be approximated by (cf. Eqs. (19) and $(20))$ :

$\Pi \simeq \frac{\left(\gamma_{\min } m_{\mathrm{e}} c^{2} \dot{n}_{ \pm}\right) R_{\gamma \gamma}}{3 \rho_{1} u_{1}^{3}}$

Consequently, Eq. (27) can be re-written:

$\widetilde{n}_{0}=\Pi \frac{u_{1}^{2}}{c^{2}} \widetilde{R}_{\gamma \gamma} \frac{(s-1)}{\gamma_{\min }^{2-s}} r$

which links $\widetilde{n}_{0}$ to $\Pi$ and $\widetilde{R}_{\gamma \gamma}$.

To close our systems of equations, we now need to link the parameters $\Pi$ and $\widetilde{R}_{\gamma \gamma}$ to $\widetilde{n}_{0}$ and $r$ using two other independent equations. This could be done by computing directly the pair creation rate, as follows. The particles accelerated in the shock cool via the IC process on the surrounding external soft photons. The photon spectrum emitted by a single particle of Lorentz factor $\gamma$ per unit time and dimensionless energy $\epsilon$ can be approximated by:

$$
\left.\frac{\mathrm{d} N_{\mathrm{ph}}}{\mathrm{d} t \mathrm{~d} \epsilon}\right|_{\gamma}=\frac{c}{R} \frac{l_{\mathrm{s}}}{\epsilon_{\mathrm{s}}} \frac{1}{\sigma_{T}} \frac{\mathrm{d} \sigma}{\mathrm{d} \epsilon}=\frac{c}{R} \frac{l_{\mathrm{s}}}{\epsilon_{\mathrm{s}}} \delta\left(\epsilon-\frac{4}{3} \gamma^{2} \epsilon_{\mathrm{s}}\right)
$$

where we have approximated the differential Compton diffusion cross section by a Dirac distribution, i.e. $\frac{\mathrm{d} \sigma}{\mathrm{d} \epsilon}=$ $\sigma_{\mathrm{T}} \delta\left(\epsilon-\frac{4}{3} \gamma^{2} \epsilon_{\mathrm{S}}\right)$

The photon spectrum emitted by a particle of initial Lorentz factor $\gamma$ cooling down to $\gamma=1$, is then given by:

$\left.\frac{\mathrm{d} N_{\mathrm{ph}}}{\mathrm{d} \epsilon}\right|_{\gamma}=\int_{\gamma}^{1} \frac{\mathrm{d} N_{\mathrm{ph}}}{\mathrm{d} t \mathrm{~d} \epsilon}\left(\gamma^{\prime}\right) \frac{\mathrm{d} t}{\mathrm{~d} \gamma^{\prime}} \mathrm{d} \gamma^{\prime}$

where the cooling rate $\frac{\mathrm{d} t}{\mathrm{~d} \gamma^{\prime}}=\frac{t_{\text {cool }}}{\gamma^{\prime}}$, with $t_{\text {cool }}$ given by Eq. (10). The integration of Eq. (30) then gives:

$\left.\frac{\mathrm{d} N_{\mathrm{ph}}}{\mathrm{d} \epsilon}\right|_{\gamma}=\left(\frac{3}{16 \epsilon_{\mathrm{s}}}\right)^{\frac{1}{2}} \epsilon^{-\frac{3}{2}}\left[1-H\left(\frac{4}{3} \gamma^{2} \epsilon_{\mathrm{s}}\right)\right]$

where $H$ is the Heaviside step function. It is now possible to compute the total photon spectrum emitted by the distribution of the particles crossing the shock per unit time and dimensionless energy $\epsilon$ :

$\frac{\mathrm{d} N_{\mathrm{ph}}^{\mathrm{tot}}}{\mathrm{d} t \mathrm{~d} \epsilon}(\epsilon)=\left.\int_{\sqrt{\frac{3 \epsilon}{4 \epsilon_{\mathrm{s}}}}}^{\infty} \dot{N}_{\text {part }}(\gamma) \frac{\mathrm{d} N_{\mathrm{ph}}}{\mathrm{d} \epsilon}\right|_{\gamma} \mathrm{d} \gamma$

where $\dot{N}_{\text {part }}(\gamma)=n_{2}\left(\gamma, x=0^{+}\right) u_{2} \pi R^{2}$ is the flux of particle crossing the shock. Consequently:

$$
\begin{aligned}
\frac{\mathrm{d} N_{\mathrm{ph}}^{\mathrm{tot}}}{\mathrm{d} t \mathrm{~d} \epsilon}(\epsilon) \simeq & \frac{\widetilde{n}_{0} l_{\mathrm{kin}}}{r} \frac{c^{2}}{u_{1}^{2}} \frac{R c}{\sigma_{T}}\left(\frac{3}{16 \epsilon_{\mathrm{s}}}\right)^{\frac{1}{2}} \epsilon^{-\frac{s+2}{2}} \frac{\gamma_{\mathrm{th}}^{1-s}}{s-1} \\
& \times \exp \left(-\frac{\gamma_{\mathrm{th}} \sqrt{\epsilon}}{\gamma_{\mathrm{c}}}\right)
\end{aligned}
$$



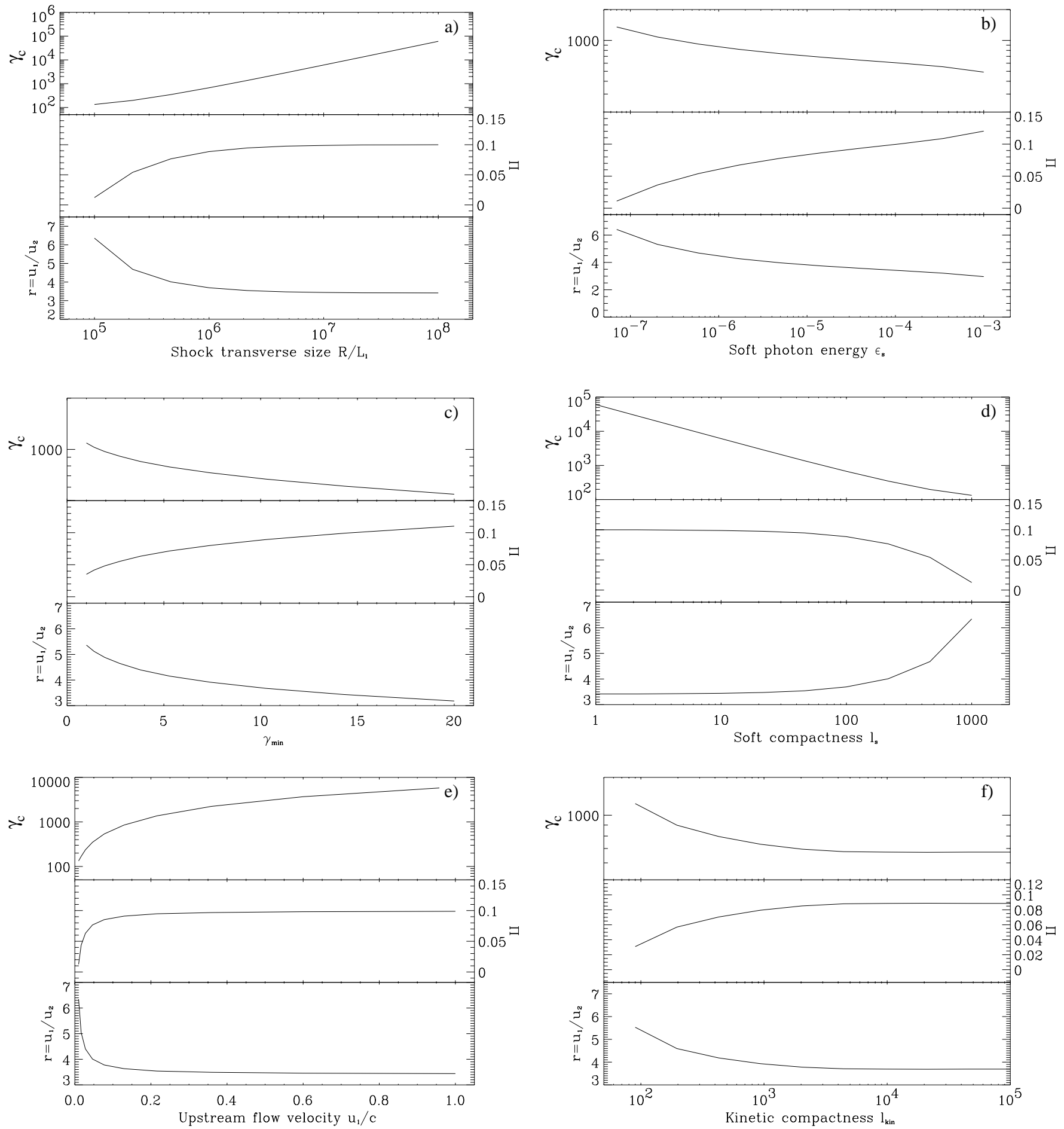

Fig. 6. Plots of $r, \Pi$ and $\gamma_{c}$ versus each parameter of the model: a) the reduced shock transverse size $\widetilde{R}$, b) the reduced soft photon energy $\left.\epsilon_{\mathrm{s}}, \mathbf{c}\right)$ the minimum Lorentz factor $\gamma_{\min }, \mathbf{d}$ ) the soft compactness $l_{\mathrm{s}}, \mathbf{e}$ ) the upstream flow velocity $u_{1} / c$, and $\mathbf{f}$ ) the flow kinetic compactness, the other parameters being fixed to $\widetilde{R}=10^{6}, \epsilon_{\mathrm{s}}=2 \times 10^{-5}, \gamma_{\min }=10, u_{1} / c=0.1, l_{\mathrm{s}}=100$ and $l_{\text {kin }}=10^{4}$.

where we define the kinetic compactness $l_{\text {kin }}=$ $\frac{\left(\rho_{1} u_{1}^{3} \pi R^{2}\right) \sigma_{T}}{R m_{\mathrm{e}} c^{3}}$. We can then deduce the pair creation rate due to the photon-photon annihilation on a length scale $R_{\gamma \gamma}$ :

$\dot{n}_{ \pm}=\frac{1}{\pi R^{2} R_{\gamma \gamma}} \int_{\epsilon=1}^{\infty} \frac{\mathrm{d} N_{\mathrm{ph}}^{\mathrm{tot}}}{\mathrm{d} t \mathrm{~d} \epsilon}(\epsilon)\left(1-\mathrm{e}^{-\tau_{\gamma \gamma}(\epsilon)}\right) \mathrm{d} \epsilon$ where $\tau_{\gamma \gamma}(\epsilon)$ is given by Eq. (7). We finally obtain a new relation between $\Pi, \widetilde{n}_{0}$ and $r$ :

$$
\begin{aligned}
\Pi= & \frac{\gamma_{\min } \widetilde{n}_{0}}{4 r} \frac{c^{2}}{u_{1}^{2}} \frac{\gamma_{\mathrm{th}}^{2-s}}{s-1} \\
& \times \int \epsilon^{-\frac{s+2}{2}} \exp \left(-\frac{\gamma_{\mathrm{th}} \sqrt{\epsilon}}{\gamma_{c}}\right)\left(1-\mathrm{e}^{-\tau_{\gamma \gamma}(\epsilon)}\right) \mathrm{d} \epsilon
\end{aligned}
$$


Finally, $\widetilde{R}_{\gamma \gamma}$ is given by Eq. (8) where:

$$
L_{\mathrm{o}}=\frac{3}{2 \sigma_{T} n_{\mathrm{ph}}(\epsilon=2)}
$$

and $n_{\mathrm{ph}}(\epsilon)=\frac{1}{\pi R^{2} c} \frac{\mathrm{d} N_{\mathrm{ph}}^{\mathrm{tot}}}{\mathrm{d} t \mathrm{~d} \epsilon}(\epsilon)$. Together with Eqs. (21), (29) and (33), this forms a system of four equations of four unknowns $\Pi, \widetilde{R}_{\gamma \gamma}, \widetilde{n}_{0}$ and $r$ which has to be verified at the equilibrium i.e. when pair pressure and hydrodynamic effects balance.

\subsection{The solutions}

The above system depends on six different parameters: $\gamma_{\min }$, the minimal Lorentz factor for the particles to be accelerated in the shock, $\epsilon_{\mathrm{s}}$, the soft photon energy (in units of $\left.m_{\mathrm{e}} c^{2}\right), u_{1}$, the upstream flow velocity, $\widetilde{R}$ the transverse size of the shock (in units of the diffusion length $L_{1}$ ), $l_{\mathrm{s}}$, the soft compactness and $l_{\text {kin }}$ the kinetic compactness.

The system is solvable in only some part of the parameter space. It always possesses two solutions: a "high pair density" one (large $\widetilde{n}_{0}$, large $\Pi$, small $r$ ) and a "low pair density" one (small $\widetilde{n}_{0}$, small $\Pi$, large $r$ ). The latter connects to the trivial solution of the problem i.e. $\Pi=0$. However, only the high pair density states are compatible with the neglecting of particles coming from the upstream flow (cf. Eq. (26)). Thus we will only focus, in the following, on this branch of solutions.

For clarity, and in order to better understand the influence of each parameter on the solutions of our problem, we have plotted in Fig. 6, the compression ratio $r$, the cut-off Lorentz factor $\gamma_{\mathrm{c}}$ and the pair luminosity $\Pi$ versus each one of these parameters, the other being fixed to values indicated in the figure caption. Some comments may be made on these different figures:

- Variation of $\widetilde{R}$ : an increase of $\widetilde{R}$ will produce an increase of $\widetilde{R}_{\gamma \gamma}$ (cf. Eq. (8)) and thus an increase of $\Pi$ and a decrease of $r$. For $\widetilde{R} \gg L_{\mathrm{o}}, \widetilde{R}_{\gamma \gamma}$ converges to $L_{\mathrm{o}}$ so that $\Pi$ and $r$ become constant (cf Fig. 6a). The cut-off Lorentz factor $\gamma_{\mathrm{c}}$ increases with $\widetilde{R}$, as expected from Eq. (11), because a constant $l_{\mathrm{s}}$ implies a decreasing photon density. For low values of $\widetilde{R}, \gamma_{\mathrm{c}}$ become smaller than $\gamma_{\mathrm{th}}$ and the decrease of the pair creation rate must be compensated by a hardening of the distribution ( $r$ increases and $\Pi$ decreases);

- Variation of $\epsilon_{\mathrm{s}}$ : when $\epsilon_{\mathrm{s}}$ increases, $\gamma_{\mathrm{th}}$ decreases, favoring the pair creation process ( $\Pi$ increases). It is compensated by a steepening of the particle distribution (i.e. $r$ decreases). Concerning $\gamma_{\mathrm{c}}$, it is a simple function of $r$ (cf. Eq. (11)), which decreases if $r$ decreases (cf. Fig. 6b);

- Variation of $\gamma_{\min }$ : since we suppose that the pairs are instantaneously accelerated to the Lorentz factor $\gamma_{\text {min }}$, the larger $\gamma_{\min }$ and the larger the pair pressure i.e. the smaller the compression ratio (cf. Fig. 6c). We recall however that for too small values of $\gamma_{\min }\left(\right.$ i.e. $\gamma_{\min } \simeq 1$ ) the annihilation of pairs is not negligible anymore and our model is no longer valid;

- Variation of $l_{\mathrm{s}}$ : the larger the soft compactness, the shorter the cooling time scale (cf. Eq. (10)) and thus the smaller $\gamma_{\mathrm{c}}$. An increase of $l_{\mathrm{s}}$ thus disfavors the pair creation process. The compression ratio has then to increase to keep the pair-hydrodynamics balance. For small $l_{\mathrm{s}}$ however, $\gamma_{\mathrm{c}}$ is very large $\left(\gg \gamma_{\mathrm{th}}\right)$ and its value becomes irrelevant. Consequently, $\Pi$ and $r$ become independent of $l_{\mathrm{s}}$, as shown in Fig. 6d;

- Variation of $u_{1}$ : when $u_{1}$ increases, $t_{\text {acc }}$ decreases and then $\gamma_{c}$ increases. The compression ratio $r$ decreases by compensation. On the other hand, for low values of $u_{1}$ (i.e. $u_{1}<0.1 c$ ), $\gamma_{\mathrm{c}}$ becomes too small, decreasing the pair creation rate and $r$ increases rapidly;

- Variation of $l_{\text {kin }}$ : for high values of $l_{\text {kin }}$, the pair density must be also high to efficiently modify the hydrodynamical profile. The pair creation process is thus saturated, meaning that the pair creation rate grows linearly with $l_{\text {kin }}$, i.e. $\Pi, r$ and $\gamma_{c}$ remain constant. However, for low values of $l_{\text {kin }}$, the pair density is so low that the pair creation optical depth becomes smaller than 1 . In compensation $r$ must increase, so $\Pi$ decreases accordingly.

\section{Discussion}

In this paper, we have seen that the launch of the pair creation process by particles accelerated by a shock embedded in a dense soft photon field could disrupt, through the increase of the associated pair pressure, the plasma flow and eventually, for too high pressure, to smooth completely the shock. By including the feedback of the hydrodynamics of the flow on the pair creation process, the system can reach, in some conditions, stationary states. Such processes may be at the origin of the high energy emission observed in compact objects where acceleration processes in dense photon field are expected to occur. We give here numerical estimates of the physical parameters relevant to this class of objects.

\subsection{High energy spectra}

As seen in the previous section, for a given set of parameters, the system may reach stationary states characterized by a compression ratio $r$. The spectral index and cut-off of the particle distribution function just after the shock $n_{2}\left(\gamma, 0^{+}\right)$are then given by Eqs. (9) and (11) respectively. Since we have assumed that these particles are cooled via the IC process (where the cooling length scale depends on the particle Lorentz factor), the emitted energy spectrum is characterized by a cut-off power law shape $F_{\mathrm{E}} \propto E^{-\alpha} \exp \left[-\left(\frac{E}{E_{\mathrm{c}}}\right)^{\frac{1}{2}}\right]($ cf. Eq. (31)) where $\alpha$ and $E_{\mathrm{c}}$ are simple functions of $s$ and $\gamma_{\mathrm{c}}$ :

$\alpha=\frac{s}{2}$ 

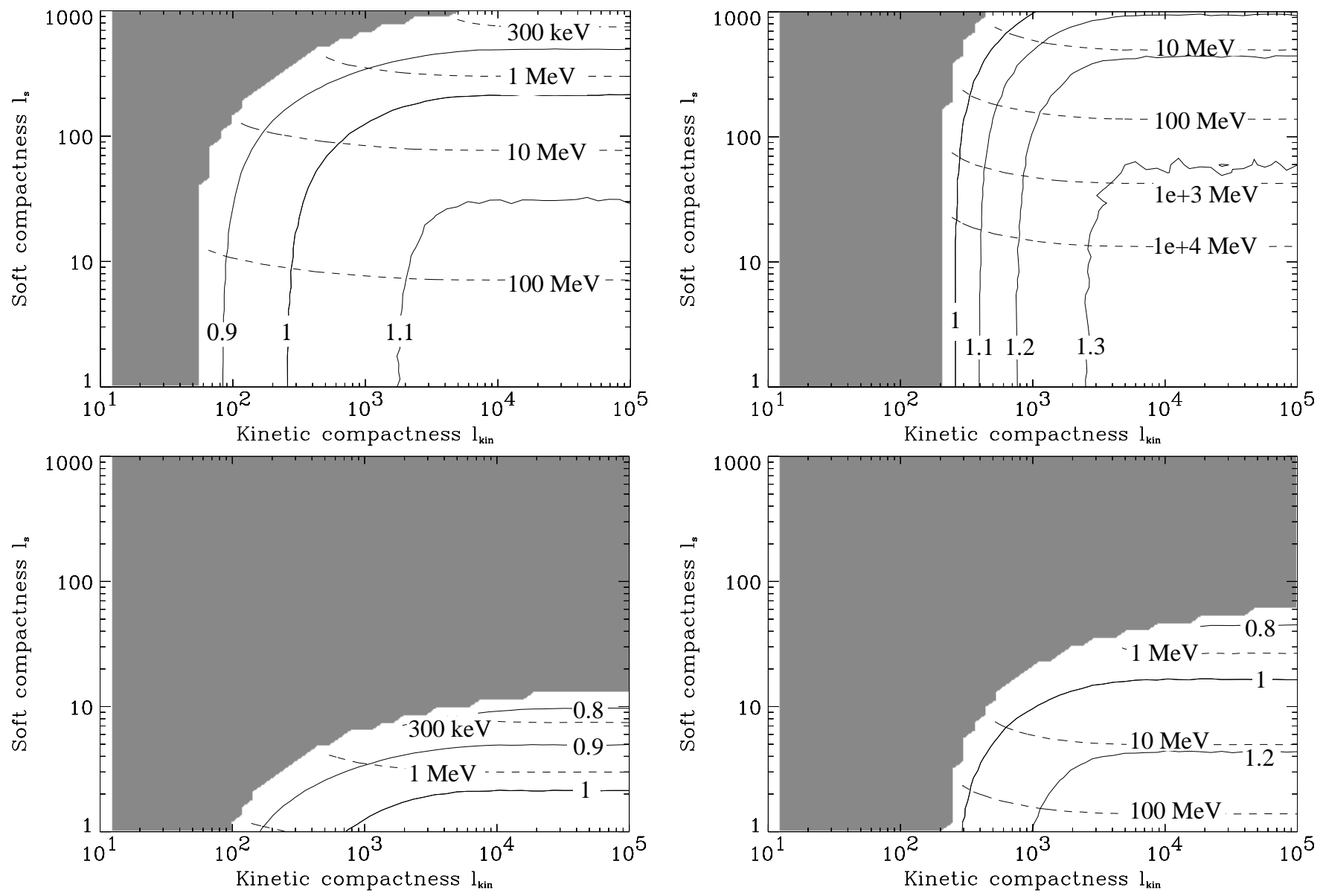

Fig. 7. Contour plots of the spectral index $\alpha$ (solid lines) and the high energy cut-off $E_{\mathrm{c}}$ in $\mathrm{keV}$ (dashed lines) of the emitted spectrum in the $\left(l_{\mathrm{s}}, l_{\text {kin }}\right)$ space. The soft photon energy is equal to $10 \mathrm{eV}$ and $600 \mathrm{eV}$ for the left and right plots respectively and $\widetilde{R}=10^{4}$ and $10^{6}$ for the lower and upper plots respectively. The other parameters have been fixed to $\gamma_{\min }=10$ and $u_{1} / c=0.1$. In the grey region there is no pair-dominated solution in stationary states.

$E_{\mathrm{c}} \simeq \frac{4}{3} \gamma_{\mathrm{c}}^{2} \epsilon_{\mathrm{s}} m_{\mathrm{e}} c^{2}$

We have reported in Fig. 7 the contour plot of $\alpha\left(l_{\mathrm{s}}, l_{\text {kin }}\right)$ and $E_{\mathrm{c}}\left(l_{\mathrm{s}}, l_{\text {kin }}\right)$ for two values of $\epsilon_{\mathrm{s}}(10$ and $600 \mathrm{eV}$ which are representative of the typical values of soft photons emitted by an accretion disk around a supermassive and stellar mass black hole respectively) and $\widetilde{R}\left(10^{4}\right.$ and $\left.10^{6}\right)$. The other parameters have been fixed to $\gamma_{\min }=10$ and $u_{1} / c=0.1$. The contours of $\alpha$ and $E_{\mathrm{c}}$ keep roughly the same shape but cover a different region of the parameter space for different parameter sets. In each figure, we can see that:

- The spectral index does not strongly vary between the harder and the softer spectra $(\Delta \alpha \simeq 0.3)$. It reaches an asymptotic plateau for low $l_{\mathrm{s}}$ and high $l_{\text {kin }}$. In these conditions, both pair density and high energy cut-off are large, so that the pair creation process is saturated. Then it can be shown that Eq. (32) reduces to a relatively simple relation between $\alpha, \gamma_{\min }$ and $\epsilon_{\mathrm{s}}$, namely:

$\gamma_{\min }^{1-2 \alpha}=\frac{1}{2 \alpha}\left(\frac{3}{4 \epsilon_{\mathrm{s}}}\right)^{1-\alpha}$.
Concerning $E_{\mathrm{c}}$, following Eqs. (11) and (36), it is inversely proportional to $l_{\mathrm{s}}^{2}$;

- The harder spectra are obtained for large values of $l_{\mathrm{s}}$ or small values of $l_{\text {kin }}$. In both cases, the pair efficiency decreases either due to a small value of $E_{\mathrm{c}}$ or a low particle density, that is a low pair production optical depth. To keep the equilibrium between the pair creation effects and the hydrodynamics of the flow, the system has to reach harder spectra to compensate this decrease of the pair creation rate;

- For small $l_{\mathrm{s}}$ (respectively large $l_{\text {kin }}$ ), $\alpha$ is independent of $l_{\mathrm{s}}$ (respectively $l_{\text {kin }}$ ). This is a direct consequence of the behavior of the compression ratio at small $l_{\mathrm{s}}$ or large $l_{\text {kin }}$ as explained in Sect. 6.2. We can note that in the case $\epsilon_{\mathrm{s}}=600 \mathrm{eV}$ and $\widetilde{R}=10^{6}$ shown in Fig. 7 the condition $\gamma_{\mathrm{c}}<m_{\mathrm{e}} c^{2} / \epsilon_{\mathrm{s}}$, needed in the Thomson regime (cf. Sect. 2.5), may not be verified in the low part of the parameter space. However, since the pair creation process is mainly triggered by particles with a Lorentz factor of the order of $\gamma_{\text {th }}\left(\ll \gamma_{c}\right)$, we do not expect the Klein-Nishina effects to strongly modify our results; 
- For too large $l_{\mathrm{s}}$ or too small $l_{\text {kin }}$, the system cannot reach sufficiently hard states to remain in equilibrium and no high pair density stationary state can exist any longer. The system can only be in the trivial pair-free state (i.e. $r=7$ and $\Pi=0$ ).

We interpret the differences between the four plots of Fig. 7 as follows. The increase of $\epsilon_{\mathrm{S}}$ favors the pair creation process. Thus, for given values of $l_{\mathrm{s}}$ and $l_{\text {kin }}$, the spectral index is larger. The high energy cut-off $E_{\mathrm{c}}$ increases mainly because of its dependence on $\epsilon_{\mathrm{s}}$ (cf. Eq. (36)), $\gamma_{\mathrm{c}}$ remaining roughly constant (cf. Eq. (11)). Concerning the variation of $\widetilde{R}$, as expected, a larger value corresponds to a more efficient acceleration (cf. Eq. (11)) and favors the pair creation process which allows a larger space parameter region for high pair density solutions.

\subsection{Annihilation line}

The presence of pairs should give a signature as an annihilation feature at $\sim 511 \mathrm{keV}$. We will show that this feature is not expected to be strong in our model. Here, we have supposed the existence in the shock region of preaccelerating processes bringing leptons the sufficient energy (i.e. $\gamma>\gamma_{\min }$ with $\gamma_{\min }$ of the order of a few, cf. Sect. 3.1.1) for resonant scattering off magnetic disturbances. Since the annihilation process occurs mainly at low energy, i.e. for particles with Lorentz factor $\gamma \simeq 1$ (cf. Coppi \& Blandford 1990), it occurs mainly far downstream, where the pairs created in the shock can cool down. The annihilation line luminosity is thus at most equal to the pair rest mass luminosity, which is itself smaller by a factor $\gamma_{\min }$ than the pair creation luminosity (Eq. (28)). As shown in Sect. 5.1, the pair luminosity $\Pi$ is itself limited and is necessarily smaller than $\sim 20 \%$ of the $\mathrm{X}$-ray $/ \gamma$-ray luminosity, i.e. $\Pi<\Pi_{\max } \simeq 0.2$ (assuming that the total kinetic energy of the upstream flow is transformed into radiation). Besides, for $\Pi \simeq 0.2$, the compression factor is very small, of the order of unity, resulting in very steep X-ray spectra. When hydrodynamic feedback is taken into account, the pair luminosity may be well below this theoretical limit of $20 \%$. An X-ray spectral photon index $\sim 2$ (as generally seen in Seyfert galaxies) requires a compression ratio $r \sim 3-4$. Such values of $r$ require values of $\Pi$ smaller than $\simeq 10 \%$ (cf. Fig 4 ).

Assuming a steady state, pairs annihilate at the same rate as they are produced. $\Pi / \gamma_{\min }$ gives then an upper limit of the annihilation line luminosity. We thus expect the luminosity of the annihilation radiation to be smaller than few percent of the total high energy radiation, which is quite compatible with the non observation of strong annihilation lines in this class of Seyfert galaxies, as shown by the best upper limit observed in Seyfert galaxies with the OSSE satellite (Johnson et al. 1997).

\subsection{Variability}

For some values of external parameters, as suggested by Fig. 7, no high pair density solution can exist in stationary states. So only the pair-free solution (i.e. with $\Pi=0$ ) exists. The system is not expected to be variable with a constant set of parameters and variability can only occur with a variation of one of them. An interesting possibility would be to consider a possible feedback of the relativistic plasma to the soft compactness. In the reillumination models for instance (Collin 1991; Henri \& Petrucci 1997), the soft photons are produced by the reprocessing of the primary X-ray emission. An increase of the pair plasma density will increase the X-ray illumination and thus the soft compactness. Figure 7 shows that in some cases, the change of $l_{\mathrm{s}}$ make the system switch to a pair-free solution which will stop the reillumination and bring the system back to pair rich solutions. Limiting cycles could thus occur. We intend to further investigate this possible effect under astrophysically relevant conditions.

\section{Conclusion}

In the present paper, we have studied the effect of pair creation, via high energy photon-photon interactions, on a shock structure, where the high energy photons are produced via the IC process by the particles accelerated by the shock itself. The problem is highly nonlinear since pairs can modify the shock profile through their pressure and, mutually, a change in the shock hydrodynamics can decrease or increase the pair production rate.

We have shown that for a given size of the pair creation region, there exists a maximal value of the pair creation rate above which the shock can no longer exist. When the hydrodynamical feedback on the pair creation process is neglected, a pair power of at most $20 \%$ of the upstream kinetic power is sufficient to kill the shock. This constraint can fall to a few percent in stationary states where pair creation and hydrodynamical effects balance. We thus do not expect the presence of strong annihilation lines. We also obtain spectral parameters in rough agreement with observations.

We suggest also a possible variability mechanism if the soft photon compactness depends itself on the pair density of the hot plasma, such as expected in reillumination models.

In the model presented here, the cooling of particles is due to the IC process on external soft photons. However, particles may also cool on soft photons they produce by the synchrotron process when spiraling around the magnetic field lines (the so-called synchrotron-self-compton process, SSC). In this case, the cooling will also depend on the particle distribution function. We may expect that the addition of the SSC process would allow us to obtain stationary states with harder spectra than those we obtained here, since the additional synchrotron cooling would be compensated by a stronger acceleration, i.e. a 
larger compression ratio $r$. The detailed study of this problem is left to future work.

Acknowledgements. We acknowledge the anonymous referee for her/his helpful comments. POP acknowledges a grant of the European Commission under contract number ERBFMRXCT98-0195 (TMR network "Accretion onto black holes, compact stars and protostars").

\section{Appendix: Particle distribution function with pair creation}

Neglecting the annihilation rate, which is only important at low energy, and asssuming that pairs are produced at a constant rate $Q$ in the region $-R_{\gamma \gamma} \leq x \leq+R_{\gamma \gamma}$ and instantaneously accelerated at $p_{\min }=\gamma_{\min } m_{\mathrm{e}} c^{2}$ (cf. Sect. 3.1.1), Eq. (13) can be rewritten (in the stationnary state) as follows:

$$
\begin{aligned}
\frac{\partial f}{\partial t}+u \frac{\partial f}{\partial x}= & \frac{1}{3} \frac{\partial u}{\partial x} p \frac{\partial f}{\partial p}+\frac{1}{p^{2}} \frac{\partial b p^{4} f}{\partial p}+\frac{\partial}{\partial x} D \frac{\partial f}{\partial x}+ \\
& Q \delta\left(p-p_{\min }\right) H\left(R_{\gamma \gamma}-|x|\right)
\end{aligned}
$$

where $H(x)$ the Heaviside function defined by

$$
\begin{aligned}
H(x) & =1 \text { for } x \geq 0 \\
& =0 \text { everywhere else. }
\end{aligned}
$$

As shown in Sect. 3.2.2, the shock thickness (which is of the order of the diffusion length) is smaller by far than any of the characteristic lengths of the problem. Consequently, when integrating Eq. (37) between $-\infty$ and $0^{+}$, we can still approximate the velocity gradient by a Dirac $\frac{\partial u}{\partial x}=$ $\left(u_{2}-u_{1}\right) \delta(x)$. We can also neglect the coolings for $x \leq 0$ for particles with a Lorentz factor $\gamma \leq \gamma_{\mathrm{c}}$ (cf. Sect. 3.1.2). The result of the integration gives:

$$
\begin{aligned}
f_{0^{+}}+\left(\frac{r-1}{3 r}\right) p \frac{\partial f_{0^{+}}}{\partial p}= & f_{1}+\frac{Q R_{\gamma \gamma}}{u_{1}} \delta\left(p-p_{\min }\right) \\
& +\frac{D}{u_{1}} \frac{\partial f_{0^{+}}}{\partial x}
\end{aligned}
$$

where $f_{0+}$ and $f_{1}$ are the distribution function in $0^{+}$and in $-\infty$ respectively. In the downstream flow, only the pair creation process will modify the distribution of particles, and the main result will be an increase of the total number of particles on a scale of the order of $R_{\gamma \gamma}$. We can thus make the following approximation:

$$
\frac{D}{u_{1}} \frac{\partial f_{0^{+}}}{\partial x} \simeq \frac{D}{u_{2}} \frac{u_{2}}{u_{1}} \frac{f_{0^{+}}}{R_{\gamma \gamma}} \simeq \frac{L_{2}}{R_{\gamma \gamma}} \frac{f_{0^{+}}}{r} .
$$

Since we have $R_{\gamma \gamma} \gg L_{2}$, this term is negligible in Eq. (38), and its integration gives:

$f_{0^{+}}(p)=q p^{-q} \int_{0}^{p}\left[f_{1}\left(p^{\prime}\right)+\frac{Q R_{\gamma \gamma}}{u_{1}} \delta\left(p^{\prime}-p_{\min }\right)\right] p^{\prime q-1} \mathrm{~d} p^{\prime}$.

With $Q=0$, one again finds the solution without pair creation. For $p>p_{\min }=\gamma_{\min } m_{\mathrm{e}} c$, the integral is almost constant. We thus find that the distribution function is a power law with a spectral index $q=\frac{3 r}{r-1}$ in any case.

\section{References}

Axford, W. I., Fillius, W., Ip, W.-H, \& Gleeson, L. J. 1976, ApJ, 210, 603

Bell, A. R. 1978, MNRAS, 182, 147

Biermann, P. L., \& Strittmatter, P. A. 1987, ApJ, 322, 643

Blandford, R. D., \& Ostriker, J. P. 1978, ApJ, 221, L29

Blandford, R. D. 1980, ApJ, 238, 410

Bregman, J. N. 1985, ApJ, 288, 32

Schlickeiser, R., Campeanu, A., \& Lerche, L. 1993, A\&A, 276, 614

Casse, F., Lemoine, M., \& Pelletier, G. 2001, submitted

Coppi, P. S., \& Blandford, R. D. 1990, MNRAS, 245, 453

Drury, L. O., \& Voelk, J. H. 1981, ApJ, 248, 344

Drury, L. O., Axford, W. I., \& Summers, D. 1982, MNRAS, 198, 833

Drury, L. O., Duffy, P., Eichler, D., et al. 1999, A\&A, 347, 370

Haardt, F., \& Maraschi, L. 1991, ApJ, 380, L51

Heavens, A. F. 1983, MNRAS, 204, 699

Heavens, A. F., \& Meisenheimer, K. 1987, MNRAS, 225, 335

Henri, G., \& P. O., Petrucci 1997, A\&A, 326, 87

Henri, G., Pelletier, G., Petrucci, P. O., \& Renaud, N. 1999, Astroparticle Phys., 11, 347

Jokipii, J. R. 1976, ApJ, 208, 900

Jones, F. C. 1994, ApJS, 90, 561

Johnson, N. 1997, American Astronomical Society Meeting, 190, 2005

Krymskii, G. F. 1977, Akademiia Nauk SSSR, Doklady, 234, 1306

Lacombe, C. 1977, A\&A, 54, 1

Lagage, P. O., \& Cesarsky, C. J. 1981, Plasma Astrophys., 317

Mushotzky, R. F., Done, C., \& Pounds, K. A. 1993, ARAA, 31,717

Nandra, K., \& Pounds, K. A. 1994, MNRAS, 268, 405

Nandra, K., George, I. M., Mushotzky, R. F., Turner, T. J., \& Yaqoob, T. 1997, ApJ, 477, 602

Nishikawa, K.-I., Sakai, J.-I., Zhao, J., Neubert, T., \& Buneman, O. 1994, ApJ, 434, 363

Parker, E. N. 1965, ApJ, 142, 1086

Pelletier, G., \& Roland, J. 1984, Ap\&SS, 100, 351

Protheroe, R. J., \& Stanev, T. 1999, Astroparticle Phys., 10, 185

Ragot, B. R., \& Schlickeiser, R. 1998, Astroparticle Phys., 9, 79

Rybicki, G. B., \& Lightman, A. P. 1979, Radiative processes in astrophysics, (New York, Wiley-Interscience),

Sambruna, R. M., Eracleous, M., \& Mushotzky, R. F. 1999 [astro-ph/9910513]

Schlickeiser, R. 1984, A\&A, 136, 227

Schneider, P., \& Kirk, J. G. 1987, ApJ, 323, L87

Skilling, J. 1975, MNRAS, 172, 557

Svensson, R. 1987, MNRAS, 227, 403

Vainio, R., \& Schlickeiser, R. 1998, A\&A, 331, 793

Vainio, R., \& Schlickeiser, R. 1999, A\&A, 343, 303

Walter, R., Orr, A., Courvoisier, T. J.-L., et al. 1994, A\&A, 285, 119

Webb, G. M., Drury, L. O., \& Biermann, P. 1984, A\&A, 137, 185

Zdziarski, A. A., \& Lightman, A. P. 1985, ApJ, 294, L79 